Douglas AL, et al. Deletion of a 197-amino-acid region in the N-terminal domain of spike protein attenuates porcine epidemic diarrhea virus in piglets. J Virol. 2017;91:e00227-17. https:/ /doi.org/10.1128/JVI.00227-17

Address for correspondence: Leyi Wang, Department of Veterinary Clinical Medicine and the Veterinary Diagnostic Laboratory, College of Veterinary Medicine, University of Illinois, Urbana, IL, 61802, USA; email: leyiwang@illinois.edu

\section{Human Case of Severe Fever with Thrombocytopenia Syndrome Virus Infection, Taiwan, 2019}

\author{
Shih-Huan Peng, ${ }^{1}$ Su-Lin Yang, ${ }^{1}$ Shih-En Tang, \\ Tzy-Chen Wang, Tung-Chien Hsu, Chien-Ling Su, \\ Meng-Yu Chen, Masayuki Shimojima, \\ Tomoki Yoshikawa, Pei-Yun Shu
}

\begin{abstract}
Author affiliations: Centers for Disease Control, Ministry of Health and Welfare, Taipei, Taiwan (S.-H. Peng, S.-L. Yang, T.-C. Wang, T.-C. Hsu, C.-L. Su, M.-Y. Chen, P.-Y. Shu); Tri-Service General Hospital, Taipei (S.-E. Tang); National Defense Medical Center, Taipei (S.-E. Tang); Institute of Aerospace and Undersea Medicine, National Defense Medical Center, Taipei (S.-E. Tang); National Institute of Infectious Diseases, Tokyo, Japan (M. Shimojima, T. Yoshikawa)
\end{abstract}

DOI: https://doi.org/10.3201/eid2607.200104

We report on a 70-year-old man with fever, leukopenia, thrombocytopenia, vomiting, malaise, dyspnea, and consciousness disturbance who was infected with severe fever with thrombocytopenia syndrome virus in northern Taiwan, 2019. This autochthonous case was confirmed by reverse transcription PCR, virus isolation, and genomic sequencing.

Severe fever with thrombocytopenia syndrome (SFTS) is a tickborne infection caused by the SFTS virus (SFTSV, also known as Huaiyangshan banyangvirus), which was identified in China in 2009 (1) and afterward in South Korea (2), Japan (3), and Vietnam

${ }^{1}$ These first authors contributed equally to this article.
(4). Since then, the number of SFTS cases in East Asia has risen rapidly. Therefore, laboratory-based surveillance of SFTS has been conducted in the routine molecular diagnosis of arboviral infections in the Taiwan Centers for Disease Control (Taiwan CDC) since 2013. We identified a patient in Taiwan with laboratory-confirmed SFTS who was originally suspected of having dengue or rickettsial infections.

In November 2019, a 70-year-old man who lived in northern Taiwan and had no travel history was admitted to the hospital with a 9-day history of fever $\left(38.8^{\circ} \mathrm{C}-39.2^{\circ} \mathrm{C}\right)$, chills, nausea, vomiting, and malaise. The patient had underlying hypertension and type 2 diabetes mellitus that was controlled without medication. At hospital admission, we noted a generalized rash over the trunk and both feet. Laboratory examinations showed that the patient had leukopenia; thrombocytopenia; abnormal prothrombin time; elevated levels of aspartate transaminase, alanine transaminase, creatinine kinase, and C-reactive protein; and diagnostic disseminated intravascular coagulation (Table). Chest radiography and chest computed tomography showed patchy consolidations and ground-glass opacities of both lungs. A few hours after admission, the patient experienced a general tonic-clonic seizure, with worsening consciousness and dyspnea. He was transferred to the intensive care unit, where intubation and ventilator support began. He also received massive blood transfusions for severe thrombocytopenia, active mucosal (oral, nasal, and gastrointestinal tract) bleeding, and disseminated intravascular coagulation. Blood and sputum cultures revealed that the patient was infected with Pseudomonas aeruginosa; he received piperacillin/ tazobactam, doxycycline, and clarithromycin as empirical therapy. Results of laboratory tests for hepatitis $\mathrm{A}$ and $\mathrm{B}$ viruses, cytomegalovirus, herpes simplex virus, adenovirus, and influenza were all negative. After the patient received a diagnosis of SFTSV infection, he received treatment with intravenous immunoglobulin for 5 days. However, his condition continued to deteriorate progressively. The patient died on day 40 after illness onset as a result of multiorgan failure. Delayed diagnosis and the presence of underlying conditions in this patient, including hypertension and diabetes mellitus, may be associated with his severe disease and death (5).

The patient often spent time on a vegetable farm in a mountainous area without wearing shoes, raising suspicions for arboviral and rickettsial infections. The hospital sent blood samples, collected from the patient before the blood transfusions on day 12 after illness onset, to the Taiwan CDC for 
Table. Laboratory findings of patient with severe fever with thrombocytopenia syndrome virus infection, Taiwan, 2019.

\begin{tabular}{|c|c|c|}
\hline Laboratory finding & Patient value & Reference range \\
\hline Leukocytes, cells/ $\mu \mathrm{L}$ & $1.550 \times 10^{3}$ & $3.9-10.6 \times 10^{3}$ \\
\hline Erythrocytes, cells/ $\mu \mathrm{L}$ & $5.550 \times 10^{6}$ & $3.9-5.4 \times 10^{6}$ \\
\hline Hemoglobin, g/dL & 16.0 & $12-16$ \\
\hline Platelets $/ \mu \mathrm{L}$ & $41 \times 10^{3}$ & $150-400 \times 10^{3}$ \\
\hline$\%$ Neutrophils & 68.4 & $42-74$ \\
\hline \% Lymphocytes & 29.7 & $25-56$ \\
\hline Aspartate transaminase, $\mathrm{U} / \mathrm{L}$ & 1,326 & $0-37$ \\
\hline Alanine transaminase, $\mathrm{U} / \mathrm{L}$ & 569 & $0-40$ \\
\hline Creatinine kinase, U/L & 1,310 & $56-224$ \\
\hline Creatinine, mg/dL & 1.7 & $0.44-1.03$ \\
\hline C-reactive protein, $\mathrm{mg} / \mathrm{L}$ & 7.8 & $<5$ \\
\hline Total bilirubin, mg/dL & 1.7 & $0.2-1.2$ \\
\hline Glucose, mg/dL & 250 & $70-100$ \\
\hline Prothrombin time, $\mathrm{s}$ & 14.4 & $6.6-11.6$ \\
\hline $\begin{array}{l}\text { Activated partial } \\
\text { thromboplastin time, s }\end{array}$ & 63.4 & 23.9-34.9 \\
\hline
\end{tabular}

laboratory diagnosis of arboviral and rickettsial diseases. Arboviral infections were detected using primer sets (Appendix Table 1, https://wwwnc. cdc.gov/EID/article/26/7/20-0104-App1.pdf) by SYBR-Green I-based real-time reverse transcription PCR (RT-PCR). In addition, we detected the SFTSV genome using SFTSV-specific primer sets targeting nonstructural protein and nucleocapsid protein genes. Results of RT-PCR and PCR for flavivirus and chikungunya virus infections, scrub typhus, murine typhus, spotted fever rickettsiae, and leptospirosis were all negative.

SFTSV was isolated from patient serum with the Vero cell line and confirmed by RT-PCR and immunofluorescence assays. SFTSV RNA remained undetected in the urine sample. The viral loads in serum continuously decreased from day 12 after disease onset and became undetectable on day 29 after disease onset (Appendix Figure 1). SuperScript III 1-step RT-PCR (http://www.thermofisher.com) identified partial small (S), medium (M), and large (L) segments of SFTSV in the serum collected on day 12 after disease onset using a different set of primers (Appendix Table 2). SFTSV has been classified into 6 different genotypes according to its genome sequence (6). Phylogenetic analyses of the partial S (1,704 bp; GenBank accession no. MN830173), M (3,340 bp; GenBank accession no. MN830174), and L (6,332 bp; GenBank accession no. MN910270) segment sequences using MEGA7 (7) using the maximum-likelihood method (Appendix Figures 2, 3) showed that the partial S, M, and L segments of SFTSV from this patient belong to genotype B and are closely related to Japanese strains. SFTSV identified in Rhipicephalus microplus ticks in central Taiwan belongs to genotype A/C (8).

All of the patient's close contacts, including 8 family members, a friend, and 60 medical personnel, were healthy and without symptoms during the monitoring period. Six mites from 2 brown rats (Rattus norvegicus) and 2 Asian house shrews (Suncus murinus) were captured in the area surrounding the residence of the patient. All the RNA samples from animals and mites showed SFTSV negative results by RT-PCR.

Although the main SFTSV tick vector, Haemaphysalis longicornis, has not been documented in Taiwan, other tick vectors, such as R. microplus and Amblyomma testudinarium, have been found in wild and domestic animals (8-10). Further studies on the identification of natural vectors and routes of transmission are needed. The presence of an emerging SFTS case highlights the need for further studies of the prevalence, geographic distribution, and surveillance of SFTSV in Taiwan.

This work was supported by grant no. MOHW108CDC-C-315-133121 from the Centers for Disease Control, Ministry of Health and Welfare, Taiwan.

\section{About the Authors}

Dr. Peng is a postdoctoral research fellow at the Center for Diagnostics and Vaccine Development, Taiwan Centers for Disease Control. His research interests include the epidemiology of rickettsial diseases and development of molecular detection methods for vectorborne infectious diseases. Dr. Yang is a senior technical specialist at the Center for Diagnostics and Vaccine Development, Taiwan Centers for Disease Control. Her research interests include the epidemiology of rickettsial diseases and development of serological detection methods for vectorborne infectious diseases.

\section{References}

1. Yu XJ, Liang MF, Zhang SY, Liu Y, Li JD, Sun YL, et al. Fever with thrombocytopenia associated with a novel bunyavirus in China. N Engl J Med. 2011;364:1523-32. http:/ / dx.doi.org/10.1056/NEJMoa1010095

2. Kim KH, Yi J, Kim G, Choi SJ, Jun KI, Kim NH, et al. Severe fever with thrombocytopenia syndrome, South Korea, 2012. Emerg Infect Dis. 2013;19:1892-4. http://dx.doi.org/10.3201/eid1911.130792

3. Takahashi T, Maeda K, Suzuki T, Ishido A, Shigeoka T, Tominaga $\mathrm{T}$, et al. The first identification and retrospective study of severe fever with thrombocytopenia syndrome in Japan. J Infect Dis. 2014;209:816-27. http:/ / dx.doi.org/ 10.1093/infdis/jit603

4. Tran XC, Yun Y, Van An L, Kim SH, Thao NTP, Man PKC, et al. Endemic severe fever with thrombocytopenia syndrome, Vietnam. Emerg Infect Dis. 2019;25:1029-31. http://dx.doi.org/10.3201/eid2505.181463

5. Zhang SF, Yang ZD, Huang ML, Wang ZB, Hu YY, Miao D, et al. Preexisting chronic conditions for fatal outcome among SFTS patients: an observational cohort study. PLoS Negl Trop Dis. 2019;13:e0007434. http://dx.doi.org/10.1371/ journal.pntd.0007434

6. Yun SM, Park SJ, Park SW, Choi W, Jeong HW, Choi YK, et al. Molecular genomic characterization of tick- and 
human-derived severe fever with thrombocytopenia syndrome virus isolates from South Korea. PLoS Negl Trop Dis. 2017;11:e0005893. http://dx.doi.org/10.1371/ journal.pntd.0005893

7. Kumar S, Stecher G, Tamura K. MEGA7: Molecular evolutionary genetics analysis version 7.0 for bigger datasets. Mol Biol Evol. 2016;33:1870-4. http://dx.doi.org/10.1093/ molbev/msw054

8. Lin TL, Ou SC, Maeda K, Shimoda H, Chan JPW, Tu WC, et al. The first discovery of severe fever with thrombocytopenia syndrome virus in Taiwan. Emerg Microbes Infect. 2020;9:14851. http://dx.doi.org/10.1080/22221751.2019.1710436

9. Tsai YL, Shyu CL, Yao CT, Lin JA. The ixodid ticks collected from dogs and other animals in Taiwan and Kinmen Island. Int J Acarol. 2012;38:110-5. http:/ / dx.doi.org/10.1080/ 01647954.2011.594812

10. Chao LL, Lu CW, Lin YF, Shih CM. Molecular and morphological identification of a human biting tick, Amblyomma testudinarium (Acari: Ixodidae), in Taiwan. Exp Appl Acarol. 2017;71:401-14. http://dx.doi.org/10.1007/ s10493-017-0119-9

Address for correspondence: Pei-Yun Shu, Vector-Borne Viral and Rickettsial Diseases Laboratory, Center for Diagnostics and Vaccine Development, Centers for Disease Control, Ministry of Health and Welfare, Taipei 11561, Taiwan; email: pyshu@cdc.gov.tw

\section{Lesions of Mycobacterium avium spp. hominissuis Infection Resembling M. bovis Lesions in a Wild Mule Deer, Canada ${ }^{1}$}

\author{
Kirsten M.F. Frayne, Brock R. Chappell, \\ Jennifer L. Davies, Bryan J. Macbeth, \\ Musangu Ngeleka, Jamie L. Rothenburger
}

Author affiliations: University of Calgary Faculty of Veterinary Medicine, Calgary, Alberta, Canada (K.M.F. Frayne, B.R. Chappell, J.L. Davies, J.L. Rothenburger); Banff National Park, Parks Canada Agency, Banff, Alberta, Canada (B.J. Macbeth); Prairie Diagnostic Services, Saskatoon, Saskatchewan, Canada (M. Ngeleka); Canadian Wildlife Health Cooperative Alberta Region, Calgary (J.L. Rothenburger)

${ }^{1}$ Preliminary results from this study were presented at the American College of Veterinary Pathologists Annual Meeting, November 9-13, 2019, San Antonio, Texas, USA
We used molecular analyses to confirm Mycobacterium avium spp. hominissuis infection in lung granulomas and pyogranulomas in the tracheobronchial lymph node in a wild mule deer in Banff, Canada. These lesions are similar to those found in M. bovis-infected animals, emphasizing the critical need for disease surveillance in wildlife populations.

DOI: https://doi.org/10.3201/eid2607.200187

Tn November 2018, a wild yearling male mule Ideer (Odocoileus hemionus) was found dead in Banff National Park, Alberta, Canada. The carcass was submitted to the Canadian Wildlife Health Cooperative Alberta Region at the University of Calgary (Calgary, Alberta, Canada) for diagnostic investigation. The University of Calgary Veterinary Sciences Animal Care Committee approved this research (AC17-0010).

Necropsy revealed that the deer had died of blunt-force trauma, presumably having been struck by a vehicle. During the necropsy, we found mineralized granulomas in the right caudal lung lobe and multifocal pyogranulomatous lymphadenitis in the tracheobronchial lymph node (Figure). We fixed lesion samples in 10\% neutral buffered formalin for 48 $\mathrm{h}$, then processed the samples by routine methods; we stained 4- $\mu m$-thick sections of paraffin-embedded tissues with hematoxylin and eosin before examination with light microscopy by an anatomic veterinary pathologist (J.L.R.), certified by the American College of Veterinary Pathologists.

Histopathology of the lung confirmed granulomatous pneumonia. Affected multifocal areas were characterized by central necrotic debris that was variably mineralized, surrounded by macrophage aggregates, multinucleated giant cells, lymphocytes, plasma cells, and variably thick fibrous capsules. Histopathology of the lymph node revealed similar multifocal areas of necrosis, variable mineralization, and infiltration of macrophages in the edges of some affected areas. Because of autolysis and freeze-thaw artifact, further characterization of the inflammatory cell population was not possible.

We designated the lymph node lesions as pyogranulomatous lymphadenitis because of the suppurative gross appearance of the lymph node combined with the microscopic presence of macrophages and mineralization revealed during histologic examination. We identified acid-fast organisms in multifocal macrophages at the margin of necrotic areas in both the lung and lymph node lesions. We submitted frozen samples to the Prairie Diagnostic Services laboratory 